\title{
An Unusual Cause of Hepatic Portal Venous
}

\section{Gas}

\author{
Nizar Talaat*, Ritu Gupta \\ Oakwood Hospital and Medical Center, Dearborn, USA \\ Email: nizartalaat@gmail.com
}

Received 29 March 2014; revised 24 April 2014; accepted 8 May 2014

Copyright (C 2014 by authors and Scientific Research Publishing Inc.

This work is licensed under the Creative Commons Attribution International License (CC BY). http://creativecommons.org/licenses/by/4.0/

(c) (i) Open Access

\begin{abstract}
A 27-years-old female with history of venous thromboembolism secondary to Lupus anticoagulant presented with sudden onset of chest pain and dyspnea. A CT angiogram of the chest showed superior vena caval (SVC) obstruction with anterior chest wall venous collaterals and hepatic portal vein gas (HPVG), which resolved without intervention on a repeated CT-scan of the abdomen. Previously, HPVG was considered to be a dangerous radiologic sign, however with the advancement in imaging technology more benign causes are being identified. This case highlights that this finding alone, if found, does not indicate aggressive management and that clinical correlation should be considered if found.
\end{abstract}

\section{Keywords}

Hepatic Portal Venous Gas, Superior Vena Cava Syndrome, Liver Imaging

\section{Introduction}

Hepatic portal venous gas (HPVG) is the presence of air in the hepatic portal venous system. This is a radiologic sign rather than an actual disease. Historically, HPVG has been associated with severe and fatal intra-abdominal pathologies that often warranted emergent surgical exploration. However, advances in abdominal imaging have led to the detection of this sign in other benign conditions that are managed conservatively.

\section{Presentation}

A 27-year-old African American female with a past medical history Crohn's Disease, multiple venous thromboembolic events secondary to Lupus anticoagulant, presented with dyspnea on exertion and non-specific chest pain of 3 days duration. She described the pain as being non-radiating and dull in nature. The patient denied any cough, diarrhea or abdominal pain. She was not taking any form of anticoagulation at the time of presentation.

"Corresponding author.

How to cite this paper: Talaat, N. and Gupta, R. (2014) An Unusual Cause of Hepatic Portal Venous Gas. Case Reports in Clinical Medicine, 3, 267-271. http://dx.doi.org/10.4236/crcm.2014.35061 
She did associate the chest pain with swelling in the upper chest, neck and face.

On physical exam, her vital signs were stable and she was in no apparent distress. Her right upper extremity and face were swollen. She also had visibly dilated veins on her anterior chest wall and upper anterior abdominal wall. On chest exam, her lungs and heart were clear to auscultation. Abdominal examination was also normal with normoactive bowel sounds and no areas of tenderness. Her laboratory value showed mild anemia and a normal liver function tests.

Because of her presentation, pulmonary embolism was suspected. A computed tomography of the chest with intravenous contrast was obtained. It showed no contrast enhancement within the intraluminal superior vena cava secondary to complete thrombosis of the SVC (Figure 1). This was associated with multiple anterior thoracic wall and anterior abdominal wall venous collaterals to the portal veins (Figure 1 and Figure 2). It also showed

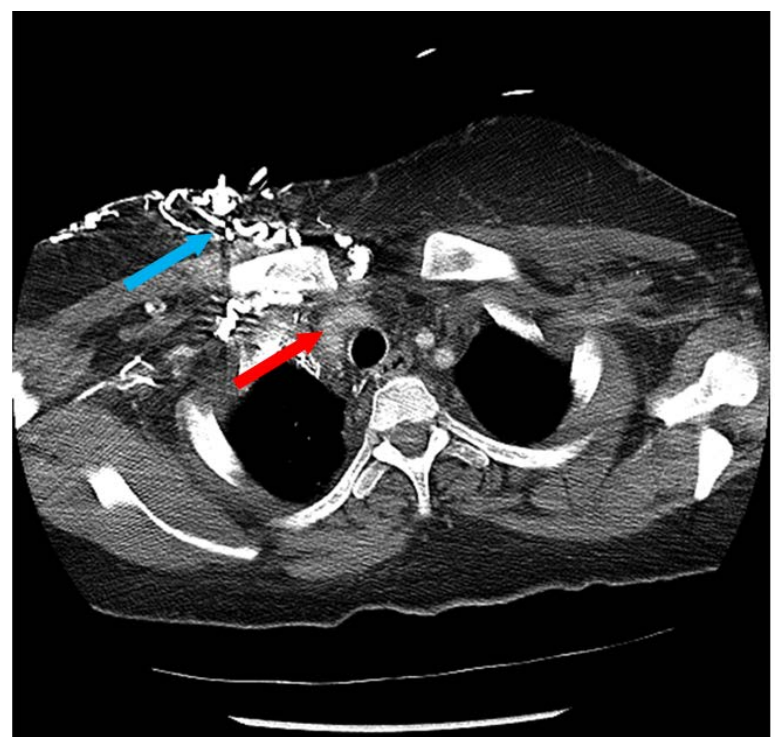

Figure 1. Complete absence of illumination of the superior vena cava (SVC) by contrast dye secondary to thrombosis (Red arrow) and extensive anterior chest wall venous collaterals (Blue arrow).

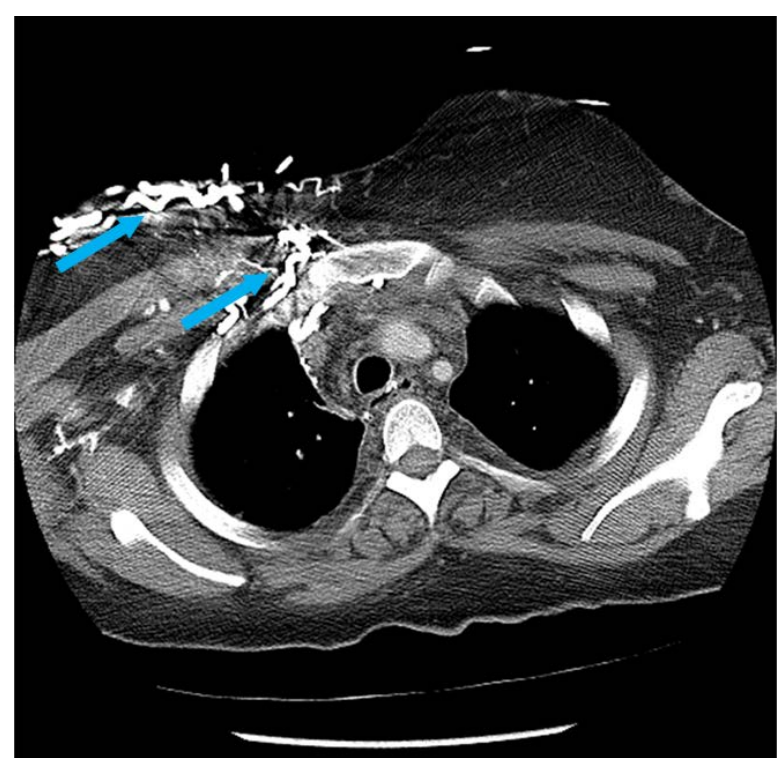

Figure 2. Extensive superficial and deep anterior chest wall venous collaterals (Blue arrows). 
migration of contrast dye and air to the portal venous system consistent with HPVG (Figure 3). The rest of her abdominal scan was normal. Due the concern of HPVG and the acute nature of her presentation the patient was placed under 24 hour observation. The following day a repeat CT scan of the abdomen revealed complete resolution of air and contrast dye from the hepatic portal venous system (Figure 4). Since the patient was clinically stable, she was discharged home on anticoagulation therapy.

\section{Discussion}

Hepatic portal venous gas (HPVG) is the presence of air in the hepatic portal venous system. This is a radiologic

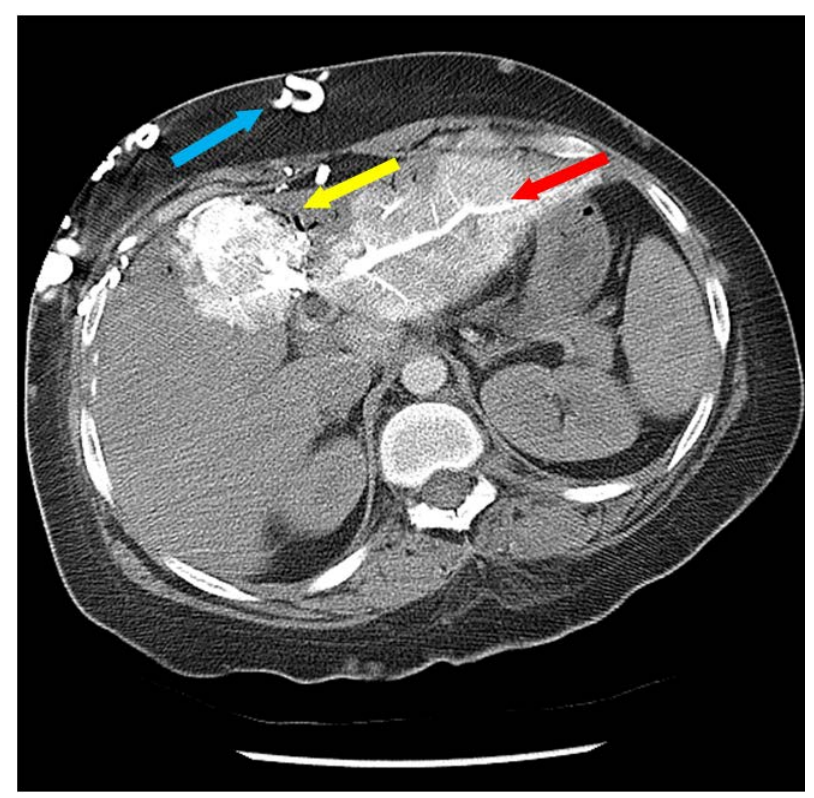

Figure 3. Contrast dye in right hepatic lobe (Red arrow) and intra hepatic venous gas (Yellow arrow). Also note anterior abdominal wall collaterals (Blue arrow).

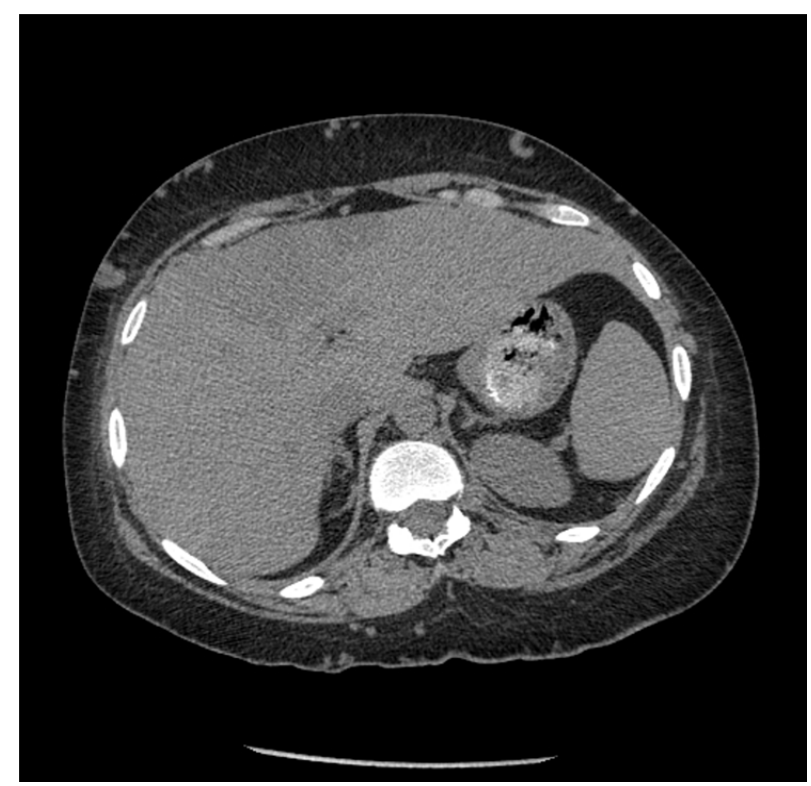

Figure 4. Complete resolution of contrast and air from the hepatic portal venous system. 
sign rather than an actual disease. Historically, HPVG has been associated with severe and fatal intra-abdominal pathologies that often warranted emergent surgical exploration. However, advances in abdominal imaging have led to the detection of this sign in other benign conditions that are managed conservatively.

Hepatic portal venous gas (HPVG) is defined in the literature as the abnormal presence of air in the hepatic venous system [1]. HPVG is not a specific disease, but rather a radiologic finding that can be visualized either by plain abdominal radiography, ultrasonography or computed tomography (CT) scan. The etiology is broad and it could occur from various benign processes to fatal abdominal diseases. Wolfe and Evens first described HPVG in infants with necrotizing enterocolitis (NEC) in the mid-fifties [2]. Following that, in 1960 Susman and Senturia described the case of HPVG in adults [3]. It has been first recognized as an ominous radiological sign associated with intra-abdominal catastrophes and high mortality. It was reported with conditions such as pneumatosis intestinalis, emphysematous pyelonephritis, necrotizing pancreatitis, inflammatory bowel disease and ischemic bowel disease and often warranted emergent surgical intervention to treat the underlying cause [4]-[9]. The exact mechanism of HPVG is not fully understood but it is thought to occur in these cases due to alteration in the intestinal wall and mucosal disruption resulting in the passage of intraluminal air into the portomesentric venous system. This in turn would lead the presence of gas-forming organisms in the portal venous system [10].

With the development of highly advanced imaging techniques, HPVG started appearing in the literature in more benign cases, usually accidently detected following different gastrointestinal procedures such as endoscopic esophageal dilatation, percutaneous liver biopsy and endoscopic retrograde cholangiopancreatography (ERCP) [11]-[15]. It is postulated that gastric dilatation during endoscopic procedures would lead to migration of air into the hepatic portal venous system [16]. In these iatrogenic cases, the presence of HPVG was usually transient and spontaneously resolved with no further intervention.

As the presence of HPVG could indicate a benign or fatal intra-abdominal, the management is usually directed towards the underlying pathology itself rather than the radiologic presence of HPVG. This would range anywhere from early surgical intervention with laparotomy to less aggressive therapy with only observation depending on the patient's clinical presentation and condition.

In the presented case, the presence of the longstanding superior vena caval obstruction has led to the development of an extensive network of collaterals within the anterior chest to the liver. During the injection of intravenous contrast dye, some air bubbles were probably present in the long intravenous tubing and the contrast syringe. The presence of venous collaterals to the liver has led to the tracking of both the dye and air to the liver.

To our knowledge, this is the first case reported of iatrogenic HPVG secondary to accidental injection of air and contrast into the peripheral venous system with the presence of collaterals to the liver. In our case, the patient has been clinically stable and therefore was carefully observed and followed up by a repeat CT scan that showed complete resolution of the air from the hepatic portal venous system.

\section{Conclusion}

Although HPVG was once considered a lethal radiologic sign that carries poor prognosis, the advanced imaging technology has led to the detection of this sign in other benign causes. There is no evidence of increased mortality associated with HVPG in these conditions. Our case highlights that this finding alone is not an indication for surgical exploration. Clinicians should be aware of benign causes of HVPG and use clinical discretion to guide appropriate management. The mainstay therapy remains to be directed towards the underlying condition itself rather than the radiologic sign.

\section{Disclosures}

None for both authors.

\section{Author Contributions}

Nizar Talaat, MD took care of the patient presented in this case report. He also prepared manuscript. Ritu Gupta, DO participated in the literature search and drafting of this manuscript.

\section{References}

[1] Griffiths, D.M. and Gough, M.H. (1986) Gas in the Hepatic Portal Veins. The British Journal of Surgery, 73, $172-176$. 
http://dx.doi.org/10.1002/bjs.1800730305

[2] Wolfe, J. and Na, E.W.A. (1955) Gas in the Portal Veins of the Liver in Infants. The American Journal of Roentgenology, Radium Therapy, and Nuclear Medicine, 74, 486.

[3] Susman, N.S.H. (1960) Gas Embolization of the Portal Venous System. The American Journal of Roentgenology, Radium Therapy, and Nuclear Medicine, 83, 847-850.

[4] Kashima, T., Ohno, Y. and Tachibana, M. (2012) Pneumatosis Intestinalis and Hepatic Portal Venous Gas in a Patient Receiving Sorafenib. International Journal of Urology: Official Journal of the Japanese Urological Association, 19, 1041-1042.

[5] Lim, J.W., Kim, K.J., Ye, B.D., et al. (2011) Enterovenous Fistulization: A Rare Complication of Crohn’s Disease. World Journal of Gastroenterology: WJG, 17, 5227-5230. http://dx.doi.org/10.3748/wjg.v17.i47.5227

[6] Siswojo, A. and Ihle, B.U. (2010) Hepatic Portal Venous Gas in a Patient with Intestinal Necrosis. Emergency Medicine Australasia: EMA, 22, 187-188.

[7] Sung, J.M., Shih, T.E. and Wu, A.B. (2010) Hepatic Portal Vein Gas Associated with Emphysematous Pyelonephritis: A Rare Association. Nephrology (Carlton), 15, 504-505. http://dx.doi.org/10.1111/j.1440-1797.2010.01209.x

[8] Wu, J.M. and Wang, M.Y. (2009) Hepatic Portal Venous Gas in Necrotizing Pancreatitis. Digestive Surgery, 26, 119120. http://dx.doi.org/10.1159/000206147

[9] Mao, Y.C., Wang, J.D. and Wang, L.M. (2009) Hepatic Portal Venous Gas Caused by Emphysematous Pyelonephritis. Clinical Gastroenterology and Hepatology: The Official Clinical Practice Journal of the American Gastroenterological Association, 7, A25.

[10] Liebman, P.R.P.M., Manny, J., Benfield, J.R. and Hechtman, H.B. (1978) Hepatic-Portal Venous Gas in Adults: Etiology, Pathophysiology and Clinical Significance. Annals of Surgery, 187, 281-287. http://dx.doi.org/10.1097/00000658-197803000-00012

[11] Lee, C.G., Kang, H.W., Song, M.K., et al. (2011) A Case of Hepatic Portal Venous Gas as a Complication of Endoscopic Balloon Dilatation. Journal of Korean Medical Science, 26, 1108-1110. http://dx.doi.org/10.3346/jkms.2011.26.8.1108

[12] Pua, U. (2010) Percutaneous Liver Biopsy: A Cause of Hepatic Portal Venous Gas. CMAJ: Canadian Medical Association Journal = Journal de l'Association medicale canadienne, 182, E861.

[13] Bobba, R.K. and Arsura, E.L. (2005) Hepatic Portal and Mesenteric Vein Gas as a Late Complication of Percutaneous Endoscopic Gastrostomy Tube Placement in an Elderly Patient. Digestive Diseases and Sciences, 50, 411-414. http://dx.doi.org/10.1007/s10620-005-1620-2

[14] Kuo, S.M., Chang, W.K., Yu, C.Y. and Hsieh, C.B. (2009) Silent Hepatic Portal Venous Gas Following Upper Gastrointestinal Endoscopy. Endoscopy, 41, E121-E122. http://dx.doi.org/10.1055/s-0029-1214657

[15] Bisceglia, M., Simeone, A., Forlano, R., Andriulli, A. and Pilotto, A. (2009) Fatal Systemic Venous Air Embolism during Endoscopic Retrograde Cholangiopancreatography. Advances in Anatomic Pathology, 16, 255-262. http://dx.doi.org/10.1097/PAP.0b013e3181aab793

[16] Nelson, A.L., Millington, T.M., Sahani, D., et al. (2009) Hepatic Portal Venous Gas: The ABCs of Management. Archives of Surgery, 144, 575-581. http://dx.doi.org/10.1001/archsurg.2009.88 